\section{P227 PERCEIVED STRESS AND SOCIOECONOMIC FACTORS ASSOCIATED WITH VAGINAL MICROBIOTA IN THE LONGITUDINAL STUDY OF VAGINAL FLORA}

${ }^{1}$ Rodman Turpin*, ${ }^{2}$ Natalie Slopen, ${ }^{1}$ Xin He, ${ }^{3}$ Courtney Robinson, ${ }^{4}$ Mark Klebanoff, ${ }^{3}$ Jacques Ravel, ${ }^{3}$ Rebecca Brotman. 'University of Maryland, College Park, Epidemiology and Biostatistics, College Park, USA; ${ }^{2}$ University of Maryland, Department of Epidemiology and Biostatistics, College Park, USA; ${ }^{3}$ University of Maryland, Institute for Genome Sciences, Baltimore, USA; ${ }^{4}$ Nationwide Children's Hospital, Columbus, USA

10.1136/sextrans-2019-sti.369

Background Lactobacillus-dominated vaginal microbiota can confer protection against STIs and high levels of stress and lower socioeconomic status are associated with increased risk for STIs. We examined whether perceived stress and demographic factors are associated with vaginal microbiota.

Methods A subsample of women $(n=1,595)$ enrolled in the Longitudinal Study of Vaginal Flora (LSVF) were utilized in this study. Vaginal microbiota was characterized by amplicon sequencing of the V3-V4 hypervariable regions of the 16S rRNA gene and clustered into community state types (CSTs). Stress in the past 30 days was measured using the Perceived Stress Scale and was categorized into quartiles. We used mixed-effects multinomial regression models accounting for within-subject associations to compare prevalence of Lactobacillus iners-dominated (CST III) and low-Lactobacillus (CST IV) versus a category of high relative abundance of other Lactobacillus spp. (CST I, II, V) across stress quartiles and demographics including education, income, race, marital status, and age.

Results In unadjusted models, the third stress quartile (reference first quartile) was associated with greater prevalence of CST III (prevalence ratio $(P R=1.15, p=0.013)$ and IV $(P R=1.22, p=0.048)$. The top quartile of stress was not statistically significant. After adjusting for covariates, results were attenuated. An education below high school level (reference high school or more) was associated with higher prevalence of CST III $(P R=1.30, p=0.002)$ and IV $(P R=1.35, p=0.002)$. Lower prevalence of CST IV was observed among participants who were White (reference Black, $P R=0.38, p<0.001$ ), and had $>\$ 3000$ monthly income (reference $<\$ 500, P R=0.80$, $\mathrm{p}=0.47)$.

Conclusion In bivariate analysis, moderate stress levels were associated with less optimal vaginal microbiota, exemplified by low abundance of Lactobacillus and L. iners-dominated microbiota. Adjustment for education and income attenuated these findings. Future studies are needed to clarify how education and income associated with vaginal microbiota, whether through access to care, sexual networks or stress affecting the body's immune response.

Disclosure No significant relationships.

\section{P228 FROM 2017 POSITIVE VOICES DATA: IS SELF-REPORTED HEALTH ASSOCIATED WITH DEPRESSIVE SYMPTOMS AMONG PEOPLE WITH HIV IN ENGLAND?}

${ }^{1}$ Natasha Ratna*, ${ }^{2}$ Meaghan Kall. ${ }^{1}$ Public Health England, HIVISTI Deparment, London, UK; ${ }^{2}$ Public Health England, HIVISTI, EQ, UK

\subsection{6/sextrans-2019-sti.370}

Background The study aims to determine whether selfreported health is associated with depressive symptoms among people with HIV.
Methods The study used cross-sectional, nationally representative survey data from people with HIV from the Positive Voices survey in 2017. Multivariable logistic regression determined associations between self-reported health and depression (defined by GHQ-12 score $\geq 4$ ), adjusted for age, employment, financial security (ability to meet basic needs), lifestyle (smoking and binge drinking), self-reported pain and comorbidities.

Results Of 2,142 MSM, 1,015 women and 583 heterosexual men, the proportion reporting good or very good health was $70 \%, 77 \%$ and $77 \%$ respectively. Prevalence of depression increased as self-reported health worsened: among MSM, 10\% reporting 'very good' health had depressive symptoms, this rose to $24 \%$ among those reporting 'good' health, 57\% (fair health) and $87 \%$ (bad health). Equivalent figures were $8 \%$, $17 \%, 45 \%, 85 \%$ for heterosexual men; and 9\%, 23\%, 59\%, $78 \%$ for women. After adjusting for other factors, the likelihood of depression increased with bad health compared to very good health among MSM (AOR 28.42, CI 14.44-55.91, $\mathrm{p}<0.0001$ ); heterosexual men (AOR 20.15, CI 4.79-84.79, $\mathrm{p}<0.0001$ ) and women (AOR 11.46, CI 3.76-34.94, $\mathrm{p}<0.0001)$. Compared to financially secure MSM, heterosexual men and women, their financially insecure counterparts were significantly more depressed: (AOR 5.02, CI 2.96-8.53, $\mathrm{p}<0.0001) ; \quad(\mathrm{AOR}$ 5.27, CI 2.12-13.14, $\mathrm{p}=0.001) ; \quad(\mathrm{AOR}$ 3.23, CI 1.79-5.83, p<0.0001). Strong association between self-reported pain and depression was observed among MSM (AOR 1.41, CI 1.07-1.84, p=0.014); heterosexual men (AOR 2.77, CI 1.42-5.42, $\mathrm{p}=0.003$ ) and women (AOR 3.79, CI 2.38-6.05, $\mathrm{p}<0.0001)$. Unemployment was strongly associated with depression among MSM (AOR 1.87, CI 1.13-3.11, $\mathrm{p}=0.022)$, but not heterosexual men $(\mathrm{p}=0.651)$ or women $(\mathrm{p}=0.288)$.

Conclusion Self-reported health is an important predictor of depressive symptoms in people with HIV, along with financial security and pain. This data can be used to inform screening tools for depression among people with HIV.

Disclosure No significant relationships.

\section{P233 GENITAL TRACT INFECTION OF WOMEN WITH AND WITHOUT TUBAL PATHOLOGY}

Svetlana Dubrovina*, Oksana Ardintseva. Rostov Medical State University, Rostov-on-Don, Russian Federation

\subsection{6/sextrans-2019-sti.371}

Background The aim of our study was to investigate the presence of Chlamydia trachomatis (CT), Neisseria gonorrhoeae (NG), Mycoplasma genitalium (MG) and non-sexual transmitted infection (STI) microorganisms in Fallopian tubes of patients with tubo-peritoneal factor of infertility diagnosed during laparoscopy or with normal tubes.

Methods 12 of 22 infertile women included in investigation had tubal-peritoneal pathology and 10 women were without tubal pathology as assessed by laparoscopy. During laparoscopy the tubes were flushed with 2-4 ml 0.9\%-saline solution, immediately after the laparoscope and auxiliary instruments were introduced. The tubes were flushed by gently grasping the tubal ampulla near the fimbria portion with atraumatic tubal forceps and introducing an epidural catheter inside the ampulla through the abdominal tubal ostium via a suprapubic 
puncture. We also took material from tubes with microbiological loop in aim to do bacteriological investigation.

Results The mean age in first group was $25 \pm 0,5$ years, in control group was $31 \pm 1$ years. Included criteria for both groups was infertility and absence of STI in endocervix. In case group all patients had pelvic inflammatory disease in anamnesis, high level of CT antibodies (MOMP and HSP), severe tubal pathology and adhesive process revealed during laparoscopy. We did not find CT, NG or any non STI microorganisms in tubes in both groups. We found twice Ureaplasma urealyticum (UU) in tubes in control group.

Conclusion The principal feature of salpingitis is extensive tissue remodeling that produces chronic sequelae such as scars and lumen obstruction and during this process the STIs and non STIs are cleared by the immune system. So, in spite of severe tubal pathology we did not find STI in Fallopian tubes. UU do not injure tubes (as we can see in control group), it could be a co-infecting pathogen, persists after antibiotics therapy and selective elimination of main pathogen.

Disclosure No significant relationships.

\section{P235 VAGINAL AND RECTAL M. GENITALIUM (MG), C. TRACHOMATIS (CT), AND N. GONORRHOEAE (GC) CO-INFECTION AMONG WOMEN IN SEATTLE, WA}

${ }^{1}$ Christine Khosropour*, ${ }^{2}$ Matthew Golden, ${ }^{3}$ Olusegun Soge, ${ }^{2}$ Lindley Barbee, ${ }^{2} J u l i a$ Dombrowski, 'Gina Leipertz, 'Anna Unutzer, 'Lisa Manhart. 'University of Washington, Epidemiology, Seattle, USA; ${ }^{2}$ University of Washington, Medicine, Seattle, USA; ${ }^{3}$ University of Washington, Global Health, Seattle, USA

10.1136/sextrans-2019-sti.372

Background Rectal CT is increasingly recognized as a common infection among women, even in the absence of anal sex. Little is known about the prevalence or epidemiologic pattern of rectal MG and rectal MG/CT/GC co-infection among women. Methods We recruited women at high risk for urogenital CT from the municipal STD clinic in Seattle, WA, 2017-2018 for a cohort study. Participants self-collected vaginal and rectal specimens for nucleic acid amplification testing (NAAT) for CT and GC. We retrospectively tested enrollment samples for vaginal and rectal MG using NAAT. We examined factors associated with rectal MG using logistic regression to calculate adjusted odds ratios (aOR) and 95\% confidence intervals (CI). Results Of 50 enrolled women, 13 (26\%) tested positive for MG. Ten (20\%) had vaginal MG and 11 (22\%) had rectal MG. Eight (16\%) women had concurrent vaginal and rectal MG, 3 (6\%) had isolated rectal MG, and 2 (4\%) had isolated vaginal MG. Among 10 women with vaginal MG, 3 (30\%) also had vaginal CT but none had vaginal GC. Of 11 women with rectal MG, 4 (36\%) also had rectal CT but none had rectal GC. Compared to women without rectal MG, women with rectal MG were more likely to be $<25$ years old $(\mathrm{aOR}=2.9 ; 95 \% \quad \mathrm{CI}=0.7-11.9)$, less likely to be White $(\mathrm{aOR}=0.22 ; 95 \% \mathrm{CI}=0.03-1.8)$, and less likely to report anal sex in the past 12 months $(\mathrm{aOR}=0.41 ; 95 \% \mathrm{CI}=0.08-2.2)$. No women with rectal MG reported anal symptoms.

Conclusion Rectal MG was common (prevalence=22\%) among women at high risk for vaginal CT, but the majority of women with MG did not have CT or GC. Rectal MG was most often identified among women with vaginal $\mathrm{MG}$, and was not significantly associated with reporting anal sex. The high prevalence of rectal MG merits further investigation to understand its natural history and clinical implications.

Disclosure No significant relationships.

\section{P237 ENZYME COMPLEXES OF ALCOHOL METABOLISM PROTECT AGAINST LIVER INJURY IN ANIMAL MODELS FED ACUTE ALCOHOL AND ANTI-HIV DRUGS}

Cheng Ji*. University of Southern California, Medicine, Los Angeles, USA

\subsection{6/sextrans-2019-sti.373}

Background A significant portion of AIDS patients under antiHIV therapies consume or abuse alcohol, which causes liver injuries. This study was to evaluate effects of eliminating blood alcohol on anti-HIV drugs and alcohol-induced liver injuries through utilizing nanoparticles of enzyme complexes of alcohol metabolism that were developed previously.

Methods The enzyme nanoparticles were intravenously injected into mouse models of acute alcohol binge or chronic alcohol and antiviral feeding in the presence of antivirals (ritonavirboosted lopinavir). Parameters for liver pathologies were examined.

Results In the acute model, the enzyme nanoparticles significantly reduced the blood alcohol concentration (BAC) within four hours compared to control. No significant effects of the anti-HIV drugs on BAC were observed in the acute alcohol binge model. Plasma alanine aminotransferase (ALT) and expression of liver TNF $\alpha$ were both significantly increased in the alcohol fed mice, which were normalized by the enzyme nanoparticles. In the presence of the antivirals, ALT was partially reduced by the enzyme nanoparticles. In the chronic alcohol feeding, alcohol induced inflammation, fatty liver and increase of ALT, which were deteriorated by the antivirals. the enzyme nanoparticles slightly reduced BAC, ALT and expression of inflammation markers of TNFo, F4/80 and IL- 6 and lipogenic factors of ACC, LXRa and SREBP1. In addition, the anti-HIV drugs potentiated alcohol induced expression of cellular organelle stress markers of CHOP, sXBP-1, ATF6 and GCP60, which were not reduced by the application of the enzyme complexes.

Conclusion Eliminating blood alcohol by the enzyme nanoparticles protects the liver against acute alcohol-induced liver injuries, and the protection is much less effective under chronic alcohol feeding or combination of alcohol and antiviral use due to severe cellular stresses in the liver. Disclosure No significant relationships. 